\title{
Next-generation sequencing of circulating tumor DNA for detection of gene mutations in lung cancer: implications for precision treatment
}

This article was published in the following Dove Press journal:

OncoTargets and Therapy

\author{
Jinhuo Lai' \\ Bin Du' \\ Yao Wang' \\ Riping $W^{\prime}$ \\ Zongyang $\mathrm{Yu}^{2}$ \\ 'Department of Oncology, Fujian \\ Medical University Union Hospital, \\ Fuzhou 35000I, Fujian Province, \\ People's Republic of China; \\ ${ }^{2}$ Department of Medical Oncology, \\ Fuzhou General Hospital of PLA, \\ Fuzhou 350025, Fujian Province, \\ People's Republic of China
}

\begin{abstract}
Background: Lung cancer remains a major global health problem, which causes millions of deaths annually. Because the prognosis is mainly determined by the stage of lung cancer, precise early diagnosis is of great significance to improve the survival and prognosis. Circulating tumor DNA (ctDNA) has been recognized as a sensitive and specific biomarker for the detection of early- and late-stage lung cancer, and next-generation sequencing (NGS) of ctDNA has been accepted as a noninvasive tool for early identification and monitoring of cancer mutations. This study aimed to assess the value of NGS-based ctDNA analysis in detecting gene mutations in lung cancer patients.
\end{abstract}

Methods: A total of 101 subjects with pathological diagnosis of lung cancer were enrolled, and blood samples were collected. ctDNA samples were prepared and subjected to NGS assays.

Results: There were 31 cases harboring 40 gene mutations, and EGFR was the most frequently mutated gene $(27.72 \%)$. In addition, there were seven cases with double mutations and one case with triple mutations, with EGFR p.T790M mutation exhibiting the highest frequency.

Conclusion: Our findings demonstrate that NGS of ctDNA is effective in detecting gene mutations in lung cancer patients, and may be used as a liquid biopsy for lung cancer, which facilitates the development of precision treatment regimens for lung cancer.

Keywords: lung cancer, next-generation sequencing, circulating tumor DNA, ctDNA, gene mutation

\section{Introduction}

Lung cancer, a malignancy characterized by uncontrolled cell growth in the lung, is a major health problem across the world. ${ }^{1,2}$ Currently, lung cancer is the most common malignancy and the leading cause of cancer-related deaths worldwide. ${ }^{3}$ According to the World Cancer Report 2014, ${ }^{4}$ there were 1.8 million patients diagnosed with lung cancer and 1.6 million deaths due to this malignancy worldwide in 2012. ${ }^{5}$ More importantly, the number of lung cancer cases is projected to be 6 million and the number of lung cancer deaths is expected to grow up to 3 million worldwide in $2035 .^{6}$

Currently, the management of lung cancer mainly includes surgery, chemotherapy, and radiotherapy, ${ }^{7}$ and targeted therapy has shown to be effective in increasing the survival. ${ }^{8-10}$ Although great advances have been achieved in these treatments, the long-term outcomes of lung cancer, which depend on the type and stage of the cancer and personal health status, remain unsatisfactory. ${ }^{11}$ The overall 5-year survival rate is estimated to be $18 \%-50 \%$ for stage I and II lung cancer patients, and $1 \%-19 \%$ for stage III and IV patients. ${ }^{12,13}$ Precise early diagnosis is therefore of great significance to improve the survival and prognosis of lung cancer. ${ }^{14}$
Correspondence: Jinhuo Lai Department of Oncology, Fujian Medical University Union Hospital, No 29 Xinquan Road, Gulou District, Fuzhou 35000I, Fujian Province, People's Republic of China

Tel +86 59l 83357896

Email ljh2252@fjmu.edu.cn

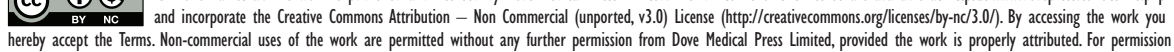
for commercial use of this work, please see paragraphs 4.2 and 5 of our Terms (https://www.dovepress.com/terms.php). 
To date, biopsy remains the gold standard for the diagnosis of lung cancer, while X-ray and computed tomography scan show great values in the screening of the malignancy. ${ }^{15}$ In the current-omics era, the multi-omics platforms have identified increasing numbers of potential biomarkers in the blood, urine, bronchial, saliva, and sputum specimens for the diagnosis of lung cancer; ${ }^{16,17}$ however, few have been applied in clinical settings. ${ }^{18}$

Circulating tumor DNA (ctDNA), which is composed of small fragments of nucleic acid that is not associated with cells or cell fragments, is found to come from tumor genome and carries tumor-specific genomic mutations. ${ }^{19}$ It has been proved that ctDNA is a sensitive and specific biomarker for the detection of early- and late-stage human malignancies including lung cancer, and can be used to monitor treatment response and detect acquired resistance to chemotherapy. ${ }^{20-22}$ In addition, the next-generation sequencing (NGS) of ctDNA has been accepted as a noninvasive tool for early identification and monitoring of cancer mutations. ${ }^{23-25}$ The major purpose of this study was to assess the value of NGS-based ctDNA in detecting gene mutations in lung cancer patients.

\section{Materials and methods}

\section{Ethical statement}

This study was approved by the Ethics Review Committee of Fujian Medical University Union Hospital (permission no FJXHYY20160027). Signed informed consent was obtained from all participants following a detailed description of the purpose of the study. All procedures of this study were performed in accordance with the international and national laws, regulations, and guidelines.

\section{Study subjects}

Lung cancer patients admitted to the Department of Oncology, Fujian Medical University Union Hospital (Fuzhou, People's Republic of China), during the period from January 2016 through December 2017, were recruited, and all diagnoses were confirmed with pathological examinations by an independent experienced pathologist. The inclusion criteria included 1) histological diagnosis of lung cancer, 2) availability of demographic and clinical data, 3) availability of blood samples for genomic analyses, and 4) provision of signed informed consent. Patients with benign lung tumors or other malignancies were excluded from the study.

\section{Blood samples, ctDNA extraction, and NGS analysis}

Blood samples were collected from each lung cancer patient, transferred to EDTA-coated tubes (BD Biosciences, San Jose,
CA, USA), and immediately centrifuged at $1,200 \times g$ at $4^{\circ} \mathrm{C}$ for 10 minutes. The supernatant was collected, transferred to $1.5 \mathrm{~mL}$ sterile Eppendorf tubes (Eppendorf, Hamburg, Germany), and centrifuged at $10,000 \times g$ at $4^{\circ} \mathrm{C}$ for 10 minutes. The prepared plasma samples (supernatant) were immediately used for ctDNA extraction or stored at $-80^{\circ} \mathrm{C}$ for the subsequent experiments.

Genomic DNA was extracted from blood samples using a DNeasy Blood \& Tissue Kit (Qiagen, Hilden, Germany) following the manufacturer's instructions. DNA concentration was quantified with a Qubit ${ }^{\circledR}$ dsDNA HS Assay Kit on a Qubit ${ }^{\circledR}$ Fluorometer (Life Technologies, Carlsbad, CA, USA), and DNA quality $\left(A_{260} / A_{280}\right.$ ratio of $\left.1.8-2.0\right)$ was measured with a NanoDrop ND-1000 Spectrophotometer (Thermo Fisher Scientific, Waltham, MA, USA). ctDNA sample was prepared, and end repair and size selection were performed according to the fragment size, followed by end adenylation. ${ }^{26}$ Multiple indexing adapters were ligated to the ends of the DNA fragments, and DNA fragments with specific adapters were used to construct the library. Following library construction, the library concentration was quantified using a Qubit ${ }^{\circledR} 2.0$ Fluorometer (Life Technologies) according to the manufacturer's instructions. The insert size of the library was detected on an Agilent 2100 Bioanalyzer (Agilent Technologies, Inc., Santa Clara, CA, USA), and the effective concentration of the library was accurately quantified.

The capture chips, designed by GeneOne Technology, Inc. (Shanghai, People's Republic of China), were used for hybrid capture of DNA samples, and effective DNA was enriched and submitted to the Illumina NextSeq ${ }^{\mathrm{TM}}$ 500 Sequencing System (Illumina, Inc., San Diego, CA, USA), which allows high-throughput paired-end sequencing (sequencing metrics: pair-end $150 \mathrm{bp}$ ) based on the effective concentration of the library and the data output requirements.

The sequencing data were mapped to the human genome reference sequences (Hg19) ${ }^{27}$ using BWA aligner. ${ }^{28}$ Local alignment optimization and variant calling were made by VarScan $2 .{ }^{29}$ Annotation of mutations was performed using the ANNOVAR software with $\mathrm{dbSNP}^{30}$ and other standard databases based on our self-designed platform.

\section{Statistical analysis}

All data were managed using Microsoft Excel 2010 (Microsoft Corporation, Redmond, WA, USA), and all statistical analyses were performed using the software SPSS version 22.0 (IBM Corporation, Armonk, NY, USA). 


\section{Results}

\section{Patients' characteristics}

A total of 101 eligible lung cancer patients were finally enrolled in the study. The subjects included 58 men and 43 women, and had a median age of 61 years (range, 35-78 years). Histological examinations revealed squamous cell carcinoma in 26 cases, adenocarcinoma in 66 cases, and small-cell carcinoma in nine cases. Table 1 shows the demographic and clinical characteristics of the study subjects.

\section{Characterization of NGS}

Capture sequencing was used to analyze ctDNA to identify genetic mutations in each sample. Sequencing yielded a mean size of $55 \mathrm{Mb}$ after filtering low-quality reads, and more than $80 \%$ of the bases had a Phred quality score of greater than 20 (error rate less than 1\%) in all cases. We achieved a mean sequencing depth of 3789 (1484-9804)×.

\section{Spectrum of gene mutations in lung cancer} Of all the plasma specimens, there were 31 specimens harboring known somatic mutations, and EGFR was the most frequently mutated gene $(27.72 \%, 28 / 101)$. Among the 66 cases with lung adenocarcinoma, there were 22, 1, and 1 cases harboring $E G F R, K R A S$, and $A L K$ mutations,

Table I Clinicopathological characteristics of the study subjects

\begin{tabular}{l|l|l}
\hline Characteristics & Value & $\%$ \\
\hline Median age (range, years) & 6 I (35-78) & - \\
Sex & 58 & \\
Male & 43 & 57.4 \\
Female & & 42.6 \\
Smoking status & 54 & \\
$\quad$ Smoker & 45 & 53.5 \\
Nonsmoker & 2 & 44.6 \\
Unknown & & 1.9 \\
Histology & 66 & \\
Adenocarcinoma & 26 & 65.4 \\
Squamous cell carcinoma & 9 & 25.7 \\
Small-cell carcinoma & & 8.9 \\
TNM stage & 1 & \\
I & 2 & 0.99 \\
II & 12 & 1.98 \\
III & 63 & 11.88 \\
IV & 23 & 62.38 \\
Unknown & & 22.77 \\
Previous treatment & 20 & \\
None & 15 & 19.80 \\
Targeted treatment & 14.85 \\
Radiochemotherapy/chemotherapy & 28 & 27.72 \\
Targeted and chemotherapy & 11 & 10.89 \\
Surgery & 2 & 1.98 \\
Surgery + targeted/radiochemotherapy & 7 & 6.93 \\
Unknown & 18 & 17.83 \\
\hline
\end{tabular}

respectively; among the 26 cases with lung squamous cell carcinoma, five cases were detected to harbor EGFR mutation and one case harbored NRAS mutation, while only one of the nine cases with small-cell lung carcinoma was found to harbor EGFR mutation (Table 2). In addition, EGFR mutation was found to occur in exon $19(\mathrm{n}=18)$, exon $20(\mathrm{n}=6)$, and exon $21(\mathrm{n}=11)$.

\section{ctDNA NGS detects multiple mutations in lung cancer}

Of the 101 lung cancer patients included in this study, there were eight cases $(7.92 \%)$ harboring more than one clinically actionable genetic alternation, including seven cases with double mutations and one case with triple mutations (Table 3). EGFR p.T790M mutation exhibited the highest frequency, followed by EGFR p.E746_A750delELREA and EGFR p.L747_T751delLREAT mutations. In addition, a case was detected to harbor double $A L K$ mutations (p.F1174C and p.G1269A).

\section{Discussion}

Currently, there are multiple approaches used to detect ctDNA. ${ }^{31}$ The conventional pyrosequencing and Sanger sequencing are the earliest tools developed to detect ctDNA; however, these two techniques suffer from problems, such as low sensitivity and failure to detect low amounts of DNA in blood samples, and are therefore not feasible for liquid biopsy. ${ }^{31}$ Digital PCR (dPCR) and amplification refractory mutation system-PCR are the most widely used assays to detect ctDNA, and these assays have shown high sensitivities and are of low costs; however, dPCR-based assays cannot be used for high-throughput detection and identification of unknown mutations. ${ }^{32} \mathrm{~A}$ search for ctDNA detection techniques with a high sensitivity, a high accuracy, a high-throughput processing ability, and a low cost is therefore urgently needed.

NGS, also known as massive parallel sequencing, has been recently developed, which allows simultaneous sequencing of millions of DNA fragments without previous sequence knowledge. ${ }^{33}$ NGS enables the detection of multiple alterations in a single test simultaneously, which has revolutionized the field of molecular diagnostics. ${ }^{34}$ In addition, NGS-based hybrid capture assays not only allow the identification of hotspot mutations but also allow the assessment of unknown alterations ${ }^{35}$ During the past decade, great advances have been made leading to improvement in reliability, sequencing chemistry, pipeline analyses, data interpretation, and costs which in turn have made NGS feasible in clinical practice today, notably in oncology. ${ }^{36}$ 
Table 2 ctDNA-based NGS assay detects gene mutations in lung cancer

\begin{tabular}{|c|c|c|c|}
\hline Gene & CDS mutation & Amino acid mutation & Number \\
\hline EGFR & c. $2573 \mathrm{~T}>\mathrm{G}$ & p.L858R & 11 \\
\hline EGFR & c. $2240 \mathrm{~T}>\mathrm{C}$ & p.L747S & 1 \\
\hline EGFR & c.2235_2249dell 5 & p.E746_A750delELREA & 8 \\
\hline EGFR & c.2236_2250dell 5 & p.E746_A750delELREA & 3 \\
\hline EGFR & c.2237_2254dell 8 & p.E746_S752>A & 1 \\
\hline EGFR & c.2238_2252dell 5 & p.L747_T75 I delLREAT & 3 \\
\hline EGFR & c.2239_2256dell 8 & p.L747_S752delLREATS & 1 \\
\hline EGFR & c.2240_2257dell 8 & p.L747_P753 $>S$ & 2 \\
\hline EGFR & c. $2369 \mathrm{C}>\mathrm{T}$ & p.T790M & 6 \\
\hline NRAS & $c .181 \mathrm{IC}>\mathrm{T}$ & P.Q6I* & 1 \\
\hline ALK & c. 352 IT $>$ G & p.FII74C & 1 \\
\hline$A L K$ & c. $3806 \mathrm{G}>\mathrm{C}$ & p.GI269A & 1 \\
\hline KRAS & $c .34 G>T$ & p.GI2C & I \\
\hline
\end{tabular}

Abbreviations: CDS, coding DNA sequence; ctDNA, circulating tumor DNA; NGS, next-generation sequencing.

The present study examined the possibility of using a targeted sequencing approach to identify gene mutations in plasma ctDNA of lung cancer patients. Here, we targeted ctDNA using a 66-gene panel to detect gene mutations in lung cancer. The overall rate of $E G F R$ mutation was $27.72 \%$ in the study subjects. In addition, the rate of $E G F R$ mutation was detected to be higher in lung adenocarcinoma than in lung squamous cell carcinoma, which was in agreement with previous studies. ${ }^{37}$

It has been shown that the highest rate of EGFR mutation is $21.9 \%-36 \%$ in lung cancer, ${ }^{38-40}$ and more than $60 \%$ of non-small-cell lung carcinomas (NSCLCs) express EGFR ${ }^{41}$ EGFR has, therefore, been identified as an important anticancer therapeutic target. ${ }^{42}$ More importantly, tyrosine kinase inhibitors (TKIs) are especially effective in cancer patients harboring activating EGFR mutations. ${ }^{43}$ Previous studies have demonstrated that EGFR-TKIs are the standard treatment for advanced NSCLC, and identification of EGFR mutation is a standard procedure during the treatment of advanced NSCLC patients. ${ }^{44-46}$ In this study, NGS-based ctDNA analysis revealed that EGFR mutation predominantly occurred in exons 19 and 21, and these mutations are the target sites of the first-generation EGFR-TKI gefitinib and erlotinib. ${ }^{47}$ T790M mutation was detected in six patients, and all exhibited complex EGFR mutations, which may be explained by the following two reasons: 1) Four out of six of them received EGFR-TKI treatment, and therefore, T790M mutation may have conferred the acquired EGFRTKI resistance in these four cases. ${ }^{48}$ 2) Our data may not reflect the real status of the overall population due to the inadequate sample size. In addition, a case was detected harboring both L858R and exon 19 deletion mutations in the $E G F R$ gene, which is rarely detected. Analysis of somatic genetic alterations in a set of 119 primary NSCLCs from Japan and the USA revealed multiple mutations in the EGFR gene; ${ }^{49}$ however, in this study, unfortunately, no novel EGFR mutations were identified. $N R A S, K R A S$, and $A L K$ mutations have been proved to be involved in the acquired resistance to TKIs in lung cancer, ${ }^{50,51}$ and $K R A S$ mutation is the second most frequently mutated drug target gene in lung cancer. ${ }^{52}$ It is reported that $K R A S$ has a mutation rate of $6.7 \%-13.5 \%$ in adenocarcinoma. ${ }^{52}$ In our study, there was only one case detected with KRAS mutation. The frequency of NRAS mutation was less than that of KRAS mutation,,$^{53}$ and a case was

Table 3 ctDNA-based NGS assay detects multiple gene mutations in lung cancer

\begin{tabular}{l|l|l|l}
\hline Sample no & Mutation \#I & Mutation \#2 & Mutation \#3 \\
\hline OGI7731004 & EGFRp.E746_A750delELREA & EGFR-p.L858R & - \\
OG 165740046 & EGFRp.E746_A750delELREA & EGFR-p.T790M & - \\
OG 170230012 & EGFRp.E746_A750delELREA & EGFR-p.T790M & - \\
OG 175710247 & EGFR-p.L747_P753>S & EGFR-p.T790M & - \\
OG165910019 & EGFR-p.L747_T75IdelLREAT & EGFR-p.L747S & EGFR-p.T790M \\
OG 173710110 & EGFR-p.L747_T75IdelLREAT & EGFR-p.T790M & - \\
OG 170270013 & ALK-p.FII74C & ALK-p.GI269A & - \\
OG 175910437 & EGFR-p.E746_A750delELREA & EGFR-p.T790M & - \\
\hline
\end{tabular}

Abbreviations: ctDNA, circulating tumor DNA; NGS, next-generation sequencing. 
identified with NRAS mutation in this study. To date, several different point mutations in the ALK tyrosine kinase domain have been found in patients with acquired resistance to the ALK-TKI crizotinib. ${ }^{54}$ In the present study, two cases were detected harboring $A L K$ mutations.

Multigene mutation is common in the cases of lung cancer. ${ }^{55}$ The frequency of double mutations, notably singlegene double mutations, is reported to be $3.13 \%-8.33 \%$ in lung cancer. ${ }^{55}$ In this study, we found that all multiple mutations occurred in a single gene, and the most frequent mutations were EGFR 19 exon deletion and T790M mutations. It has been shown that EGFR 19 exon deletion mutation and L858R mutation are the target sites of the first-generation EGFR-TKIs, and EGFR p.T190M mutation is involved in resistance to the first-generation EGFR-TKIs. ${ }^{47}$ In the current study, the seven cases harboring double EGFR mutations had rapid clinical progression, of which six were detected with EGFR-TKI resistance mutations.

The present study had some limitations. Firstly, only a total of 101 subjects were enrolled in this study, which represents a small sample size. Further studies with a much larger sample size are required to evaluate the potential of ctDNA-based NGS assay as a valuable tool for diagnosis and treatment of lung cancer. Secondly, it is reported that the gene mutations associated with lung cancer may vary with respect to ethnicity, race, culture, and geographic regions. ${ }^{49,56}$ Due to the small sample size, subgroup analyses were not conducted to investigate gene mutations in lung cancer patients with different ethnic and geographic backgrounds.

In summary, the results of the present study demonstrate that ctDNA panel sequencing can be used for lung cancer gene mutation identification, which highlights the potential to use peripheral blood samples as noninvasive biopsy specimens for lung cancer. More importantly, our findings may facilitate the development of precision treatment regimens for lung cancer.

\section{Acknowledgment}

This study was funded by the grant from Fujian Provincial Key Sci \& Tech Program (grant no 2012I0003).

\section{Disclosure}

The authors report no conflicts of interest in this work.

\section{References}

1. Hoffman PC, Mauer AM, Vokes EE. Lung cancer. Lancet. 2000;355(9202): 479-485.
2. Fitzmaurice C, Allen C, Barber RM, et al. Global Burden of Disease Cancer CollaborationGlobal, Regional, and National Cancer Incidence, Mortality, Years of Life Lost, Years Lived With Disability, and Disability-Adjusted Life-years for 32 Cancer Groups, 1990 to 2015: A Systematic Analysis for the Global Burden of Disease Study. JAMA Oncol. 2017;3(4):524-548.

3. Siegel RL, Miller KD, Jemal A. Cancer statistics, 2018. CA Cancer J Clin. 2018;68(1):7-30.

4. WHO. World Cancer Report 2014. Geneva: World Health Organization; 2014.

5. Torre LA, Siegel RL, Jemal A. Lung Cancer Statistics. Adv Exp Med Biol. 2016;893:1-19.

6. Didkowska J, Wojciechowska U, Mańczuk M, Łobaszewski J. Lung cancer epidemiology: contemporary and future challenges worldwide. Ann Transl Med. 2016;4(8):150.

7. Lemjabbar-Alaoui H, Hassan OU, Yang YW, Buchanan P. Lung cancer: Biology and treatment options. Biochim Biophys Acta. 2015; 1856(2):189-210.

8. Sun S, Schiller JH, Spinola M, Minna JD. New molecularly targeted therapies for lung cancer. J Clin Invest. 2007;117(10):2740-2750.

9. Larsen JE, Cascone T, Gerber DE, Heymach JV, Minna JD. Targeted therapies for lung cancer: clinical experience and novel agents. Cancer J. 2011;17(6):512-527.

10. Onn A, Herbst RS. Molecular targeted therapy for lung cancer. Lancet. 2005;366(9496):1507-1508.

11. Hirsch FR, Scagliotti GV, Mulshine JL, et al. Lung cancer: current therapies and new targeted treatments. Lancet. 2017;389(10066):299-311.

12. van Meerbeeck JP, Fennell DA, de Ruysscher DK. Small-cell lung cancer. Lancet. 2011;378(9804):1741-1755.

13. Goldstraw P, Ball D, Jett JR, et al. Non-small-cell lung cancer. Lancet. 2011;378(9804):1727-1740.

14. Nakajima T, Yasufuku K. Early lung cancer: methods for detection. Clin Chest Med. 2013;34(3):373-383.

15. Sardi AH, Islam S. Early lung cancer detection, mucosal, and alveolar imaging. Curr Opin Pulm Med. 2016;22(3):271-280.

16. Vargas AJ, Harris CC. Biomarker development in the precision medicine era: lung cancer as a case study. Nat Rev Cancer. 2016;16(8): 525-537.

17. Hassanein M, Callison JC, Callaway-Lane C, Aldrich MC, Grogan EL, Massion PP. The state of molecular biomarkers for the early detection of lung cancer. Cancer Prev Res. 2012;5(8):992-1006.

18. Mazzone PJ, Sears CR, Arenberg DA, et al. Evaluating Molecular Biomarkers for the Early Detection of Lung Cancer: When Is a Biomarker Ready for Clinical Use? An Official American Thoracic Society Policy Statement. Am J Respir Crit Care Med. 2017;196(7):e15-e29.

19. Alix-Panabières $C$, Schwarzenbach $H$, Pantel K. Circulating tumor cells and circulating tumor DNA. Annu Rev Med. 2012;63:199-215.

20. Bettegowda C, Sausen M, Leary RJ, et al. Detection of circulating tumor DNA in early- and late-stage human malignancies. Sci Transl Med. 2014;6(224):224ra24.

21. Gray ES, Rizos H, Reid AL, et al. Circulating tumor DNA to monitor treatment response and detect acquired resistance in patients with metastatic melanoma. Oncotarget. 2015;6(39):42008-42018.

22. Pereira E, Camacho-Vanegas O, Anand S, et al. Personalized circulating tumor DNA biomarkers dynamically predict treatment response and survival In gynecologic cancers. PLoS One. 2015;10(12):e0145754.

23. Aravanis AM, Lee M, Klausner RD. Next-generation sequencing of circulating tumor DNA for early cancer detection. Cell. 2017;168(4): 571-574.

24. Hou H, Yang X, Zhang J, et al. Discovery of targetable genetic alterations in advanced non-small cell lung cancer using a next-generation sequencing-based circulating tumor DNA assay. Sci Rep. 2017;7(1): 14605.

25. Thompson JC, Yee SS, Troxel AB, et al. Detection of therapeutically targetable driver and resistance mutations in lung cancer patients by next-generation sequencing of cell-free circulating tumor DNA. Clin Cancer Res. 2016;22(23):5772-5782. 
26. Mouliere F, Robert B, Arnau Peyrotte E, et al. High fragmentation characterizes tumour-derived circulating DNA. PLoS One. 2011;6(9): e23418.

27. Lander ES, Linton LM, Birren B, et al. International Human Genome Sequencing Consortium. Initial sequencing and analysis of the human genome. Nature. 2001;409(6822):860-921.

28. Li H, Ruan J, Durbin R. Mapping short DNA sequencing reads and calling variants using mapping quality scores. Genome Res. 2008;18(11): 1851-1858.

29. Koboldt DC, Chen K, Wylie T, et al. VarScan: variant detection in massively parallel sequencing of individual and pooled samples. Bioinformatics. 2009;25(17):2283-2285.

30. Yang H, Wang K. Genomic variant annotation and prioritization with ANNOVAR and wANNOVAR. Nat Protoc. 2015;10(10):1556-1566.

31. Ma M, Zhu H, Zhang C, Sun X, Gao X, Chen G. "Liquid biopsy"ctDNA detection with great potential and challenges. Ann Transl Med. 2015;3(16):235.

32. Wang W, Song Z, Zhang Y. A Comparison of ddPCR and ARMS for detecting EGFR T790M status in ctDNA from advanced NSCLC patients with acquired EGFR-TKI resistance. Cancer Med. 2017;6(1): $154-162$.

33. McDermott U. Next-generation sequencing and empowering personalised cancer medicine. Drug Discov Today. 2015;20(12):1470-1475.

34. Goodwin S, McPherson JD, Mccombie WR. Coming of age: ten years of next-generation sequencing technologies. Nat Rev Genet. 2016;17(6): $333-351$.

35. Reuter JA, Spacek DV, Snyder MP. High-throughput sequencing technologies. Mol Cell. 2015;58(4):586-597.

36. Kamps R, Brandão RD, Bosch BJ, et al. Next-Generation sequencing in oncology: genetic diagnosis, risk prediction and cancer classification. Int J Mol Sci. 2017;18(2):E308.

37. Ellison G, Zhu G, Moulis A, Dearden S, Speake G, McCormack R. EGFR mutation testing in lung cancer: a review of available methods and their use for analysis of tumour tissue and cytology samples. J Clin Pathol. 2013;66(2):79-89.

38. Martínez-Navarro EM, Rebollo J, González-Manzano R, et al. Epidermal growth factor receptor (EGFR) mutations in a series of non-smallcell lung cancer (NSCLC) patients and response rate to EGFR-specific tyrosine kinase inhibitors (TKIs). Clin Transl Oncol. 2011;13(11): 812-818.

39. Huang SF, Liu HP, Li LH, et al. High frequency of epidermal growth factor receptor mutations with complex patterns in non-small cell lung cancers related to gefitinib responsiveness in Taiwan. Clin Cancer Res. 2004;10(24):8195-8203.

40. Zhou Y, Yang Y, Yang C, et al. Epidermal growth factor receptor (EGFR) mutations in non-small cell lung cancer (NSCLC) of Yunnan in southwestern China. Oncotarget. 2017;8(9):15023-15033.

41. Gazdar AF. Activating and resistance mutations of EGFR in non-smallcell lung cancer: role in clinical response to EGFR tyrosine kinase inhibitors. Oncogene. 2009;28(Suppl 1):S24-S31.
42. Seymour LK. Epidermal growth factor receptor as a target: recent developments in the search for effective new anti-cancer agents. Curr Drug Targets. 2001;2(2):117-133.

43. Ranson M. Epidermal growth factor receptor tyrosine kinase inhibitors. Br J Cancer. 2004;90(12):2250-2255.

44. da Cunha Santos G, Shepherd FA, Tsao MS. EGFR mutations and lung cancer. Annu Rev Pathol. 2011;6:49-69.

45. Roengvoraphoj M, Tsongalis GJ, Dragnev KH, Rigas JR. Epidermal growth factor receptor tyrosine kinase inhibitors as initial therapy for non-small cell lung cancer: focus on epidermal growth factor receptor mutation testing and mutation-positive patients. Cancer Treat Rev. 2013; 39(8):839-850

46. Yamaoka T, Ohba M, Ohmori T. Molecular-targeted therapies for epidermal growth factor receptor and its resistance mechanisms. Int $J$ Mol Sci. 2017;18(11):E2420.

47. Morgillo F, della Corte CM, Fasano M, Ciardiello F. Mechanisms of resistance to EGFR-targeted drugs: lung cancer. ESMO Open. 2016; 1(3):e000060

48. Kim Y, Ko J, Cui Z, et al. The EGFR T790M mutation in acquired resistance to an irreversible second-generation EGFR inhibitor. $\mathrm{Mol}$ Cancer Ther. 2012;11(3):784-791.

49. Paez JG, Jänne PA, Lee JC, et al. EGFR mutations in lung cancer: correlation with clinical response to gefitinib therapy. Science. 2004; 304(5676):1497-1500.

50. Ohashi K, Sequist LV, Arcila ME, et al. Lung cancers with acquired resistance to EGFR inhibitors occasionally harbor BRAF gene mutations but lack mutations in KRAS, NRAS, or MEK1. Proc Natl Acad Sci U S A. 2012;109(31):E2127-E2133.

51. Kosaka T, Yamaki E, Mogi A, Kuwano H. Mechanisms of resistance to EGFR TKIs and development of a new generation of drugs in non-smallcell lung cancer. J Biomed Biotechnol. 2011;2011:165214-165217.

52. Karachaliou N, Mayo C, Costa C, et al. KRAS mutations in lung cancer. Clin Lung Cancer. 2013;14(3):205-214.

53. Ohashi K, Sequist LV, Arcila ME, et al. Characteristics of lung cancers harboring NRAS mutations. Clin Cancer Res. 2013;19(9):2584-2591.

54. Lovly CM, Heuckmann JM, de Stanchina E, et al. Insights into ALKdriven cancers revealed through development of novel ALK tyrosine kinase inhibitors. Cancer Res. 2011;71(14):4920-4931.

55. Su J, Zhang XC, An SJ, et al. Detecting the spectrum of multigene mutations in non-small cell lung cancer by Snapshot assay. Chin J Cancer. 2014;33(7):346-350.

56. Ellison G, Zhu G, Moulis A, Dearden S, Speake G, McCormack R. EGFR mutation testing in lung cancer: a review of available methods and their use for analysis of tumour tissue and cytology samples. J Clin Pathol. 2013;66(2):79-89.
OncoTargets and Therapy

\section{Publish your work in this journal}

OncoTargets and Therapy is an international, peer-reviewed, open access journal focusing on the pathological basis of all cancers, potential targets for therapy and treatment protocols employed to improve the management of cancer patients. The journal also focuses on the impact of management programs and new therapeutic agents and protocols on
Dovepress

patient perspectives such as quality of life, adherence and satisfaction. The manuscript management system is completely online and includes a very quick and fair peer-review system, which is all easy to use. Visit http://www.dovepress.com/testimonials.php to read real quotes from published authors. 